complete replacement of the aortic arch, the intrathoracic isolation of the proximal segment of the arch vessels is necessary anyway. The preparation of a somewhat longer proximal segment of the common carotid artery is not time-consuming and normally does not require an additional surgical access. Even in cases where the intrathoracic cannulation is not possible, the carotid artery is better accessible than the axillary artery. Furthermore, its wall is not as delicate or as easily injured. Interruption of the arterial flow during crossclamping can be avoided by using an intraluminal shunt; however, this should only be rarely necessary, as the anastomosis with the graft usually takes only a few minutes.

Interest in the common carotid artery as an arterial cannulation site has recently increased, although in most cases it was only used for additional perfusion of the upper half of the body with simultaneous cannulation of the femoral artery. ${ }^{8}$

The cannulation technique of the left common carotid artery described offers a simple installation of cardiopulmonary bypass with antegrade flow and mostly without the necessity for a separate access in addition to the median sternotomy. Furthermore, it offers the possibility of selective cerebral perfusion during extensive operations on the aortic arch. Thanks to the absence of complete interruption of the cerebral perfusion, it minimizes the risk of air embolism and avoids the disadvantages of deep hypothermia.

\section{References}

1. Urbanski PP. Valve-preserving aortic root reconstruction (Letter). J Thorac Cardiovasc Surg. 2001;121:1220.

2. Urbanski PP. Replacement of the ascending aorta and aortic valve with a valved stentless composite graft. Ann Thorac Surg. 1999;67:1501-2.

3. Urbanski PP. Complete aortic root replacement in patients with small aortic annulus. Ann Thorac Surg. 2002;73:725-8.

4. Sabik JF, Lytle BW, McCarthy PM, Cosgrove DM. Axillary artery: an alternative site of arterial cannulation for patients with extensive aortic and peripheral vascular disease. J Thorac Cardiovasc Surg. 1995;109: 885-91.

5. Whitlark JD, Scott MG, Sutter FP. Axillary artery cannulation in acute aortic dissection. Ann Thorac Surg. 2000;69:1127-9.

6. Imanaka K, Kyo S, Tanabe H, Ohnchi H, Asano H, Yokote Y. Fatal intraoperative dissection of the innominate artery due to perfusion through the right axillary artery. J Thorac Cardiovasc Surg. 2000;120: 405-6.

7. Miyatake T, Matsui Y, Suto Y, Imamura M, Shiiya N, Murashita T, Yasuda K. A case of intraoperative aortic dissection caused by cannulation into an axillary artery. J Cardiovasc Surg (Torino). 2001;42:80911.

8. Neri E, Massetti M, Barabesi L, et al. Extrathoracic cannulation of the left common carotid artery in thoracic aorta operations through a left thoracotomy: preliminary experience in 26 patients. $J$ Thorac Cardiovasc Surg. 2002;123:901-10.

\title{
Giant atherosclerotic aneurysm of the left anterior descending artery
}

\author{
Nawwar Al Attar, FRCS, FETCS, ${ }^{a}$ Jean-Louis Sablayrolles, MD, ${ }^{\text {b }}$ and Patrick Nataf, MD, ${ }^{\text {a }}$ St Denis, France
}

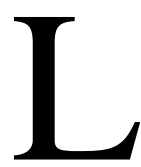

arge coronary aneurysms are mostly due to Kawasaki's disease. We report a case of giant atherosclerotic aneurysm of the left anterior descending artery (LAD) discovered incidentally in a patient with no previous coronary history. Multislice computed tomography (CT) scan of the heart gave excellent iconography, allowing accurate diagnosis and differentiating it from an aneurysm of the pulmonary artery.

\section{Case History}

A 67-year-old man of Algerian descent with no significant medical history presented with sudden onset ataxia and vertigo. The patient was overweight, afebrile, and ataxic. Blood pressure was 140/85

\footnotetext{
From the Departments of Cardiac Surgery ${ }^{\mathrm{a}}$ and Radiology, ${ }^{\mathrm{b}}$ Centre Cardiologique du Nord, St Denis, France.

Received for publication March 6, 2003; accepted for publication March 12, 2003.

Address for reprints: Patrick Nataf, MD, Department of Cardiac Surgery, Centre Cardiologique du Nord, 32-36 rue des Moulins Gémeaux, 93200, St Denis, France (E-mail: natafp@wanadoo.fr).

J Thorac Cardiovasc Surg 2003;126:888-90

Copyright (C) 2003 by The American Association for Thoracic Surgery $0022-5223 / 2003 \$ 30.00+0$

doi:10.1016/S0022-5223(03)00610-X
}

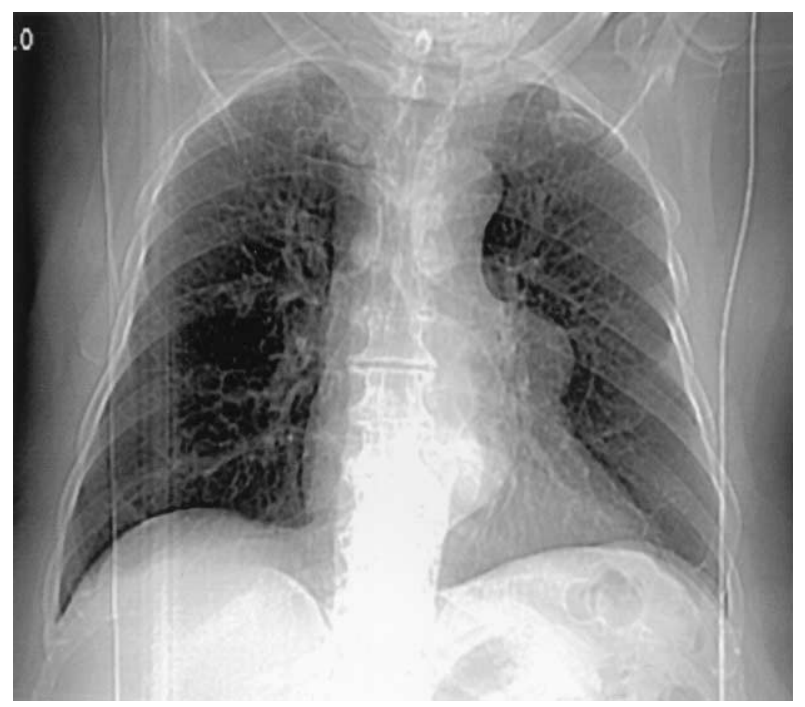

Figure 1. Chest radiograph showing left hilar mass.

$\mathrm{mm} \mathrm{Hg}$ and heart rate was regular. Brain CT scan showed left cerebellar infarction. As part of his workup, a posteroanterior chest radiograph showed a voluminous left hilar mass (Figure 1). 

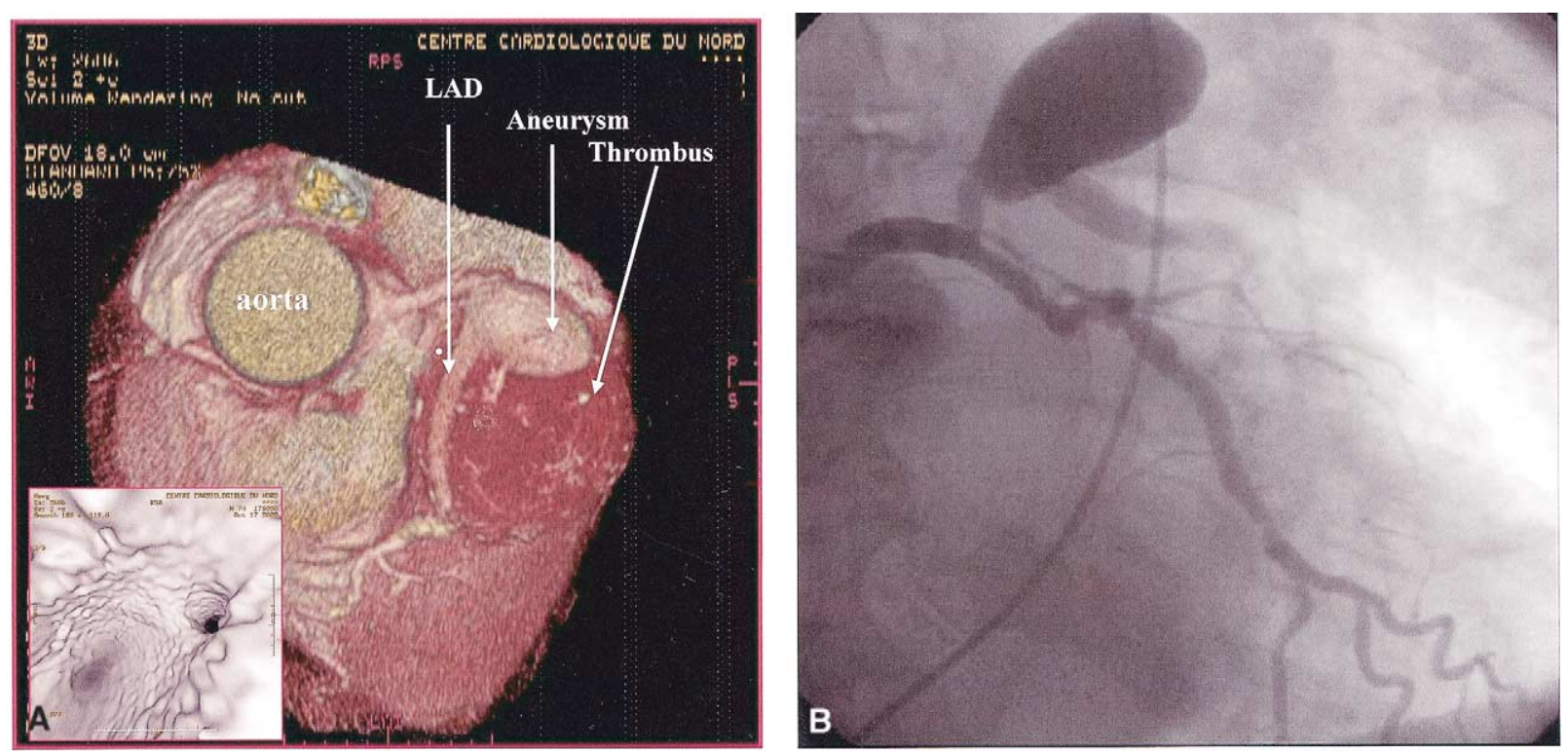

Figure 2. A, Multislice CT scan of the heart (view from above). The aneurysm is clearly shown originating from the LAD. It is composed of a large thrombus surrounding a patent inner channel. Inset is virtual endoscopy of the LAD and aneurysm. B, Coronary angiography of left coronary artery demonstrating the circulating channel of the LAD aneurysm.

Thoracic CT angioscan (GE Lightspeed ${ }^{16}$, GE Medical Systems, Chicago. Ill) demonstrated aneurysm of the proximal LAD of $60 \mathrm{~mm}$ diameter, in close proximity to the pulmonary artery (Figure 2, A). The patient was otherwise totally asymptomatic and his electrocardiogram was normal. Echocardiography confirmed the presence of an oval mass adjacent to the pulmonary artery. There were no signs of endocarditis. Coronary angiography (Figure 2, $B$ ) showed the large proximal LAD aneurysm and significant stenoses of the LAD, with diagonal and circumflex arteries. Doppler imaging demonstrated occlusion of the left internal carotid artery. Leukocyte count was normal, as were markers of inflammatory syndrome. Antiphospholipid antibodies were not detected. Serology for Lyme disease, syphilis, AIDS, and hepatitis B and C were all negative. Biopsy of the temporal artery showed no signs of polyarteritis.

The patient was operated on 2 months after his stroke under cardiopulmonary bypass for excision of the aneurysm, which contained a large thrombus (Figure 3 and insert) plus grafting of the LAD and diagonal and marginal arteries. The excised portion of the LAD was replaced by a saphenous graft.

The patient left the surgical ward after 7 days with persistent minor ataxia. Culture of the excised aneurysm gave no growth, and histopathologic examination showed atherosclerotic changes and absence of inflammatory infiltrate in the arterial wall.

\section{Discussion}

Giant coronary artery aneurysms are rare. The most common cause is Kawasaki's disease within its classical geographic location. ${ }^{1,2}$ Although atherosclerosis is the responsible pathology in this patient, it characteristically produces small aneurysms and is associated with risk factors for coronary atherosclerosis.

Most patients are asymptomatic, but manifestations of myocar-

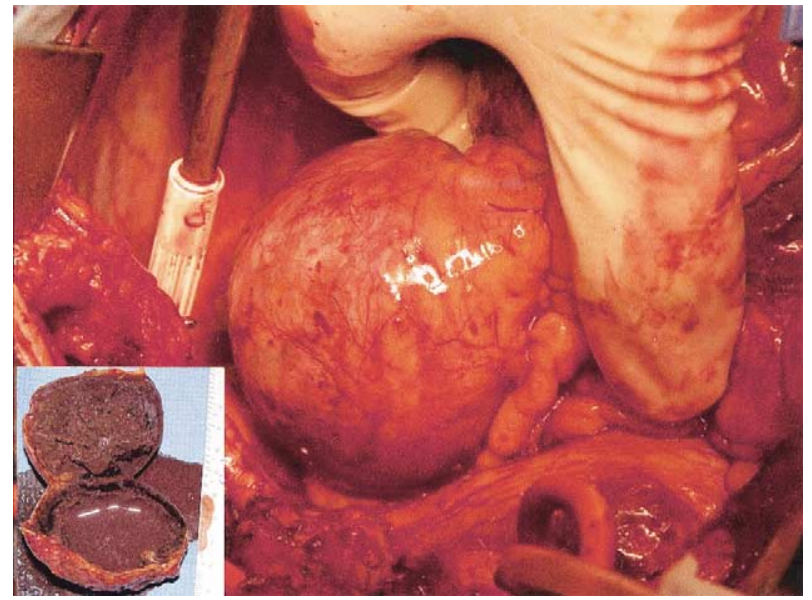

Figure 3. Operative view of the giant LAD aneurysm. Inset showed large thrombus in sectioned aneurysm.

dial ischemia may occur. Other complications include rupture, thromboembolic phenomenon, and more rarely fistulation into one of the cardiac chambers. ${ }^{3}$ Indeed, it was the cerebellar stroke that heralded the signs of atherosclerosis in our patient. Although coronary angiography is the gold standard diagnostic examination, multislice CT scan established this diagnosis and was able to differentiate the coronary aneurysm from an aneurysm of the pulmonary artery by "peeling" it from adjacent structures. With virtual endoscopy the cavity of the aneurysm was explored and shown to be occupied by a large thrombus (Figure 2, $A$ insert).

The left coronary trunk is a rare localization with little more than 30 cases described in the literature. To avoid rupture of the 
aneurysm, resection and replacement of the aneurysmal LAD with venous graft between the cut ends associated with triple bypass of the LAD and diagonal and marginal arteries with the mammary arteries crafted in a Y graft was undertaken. The size and site of the described aneurysm make it quite exceptional; furthermore, we believe that it is one of the largest atherosclerotic aneurysms reported in the medical literature. ${ }^{4,5}$

\section{References}

1. Koizumi J, Izumoto H, Ohsawa A, Ishibashi K, Ishihara K, Kawazoe K. Giant coronary artery aneurysm in diagonal artery; report of a case. Kyobu Geka. 2002;55:793-5.
2. Nakamura Y, Yashiro M, Oki I, Tanihara S, Ojima T, Yanagawa H. Giant coronary aneurysms due to Kawasaki disease: a case-control study. Pediatr Int. 2002;44:254-8.

3. Shrivastava V, Akowuah E, Cooper GJ. Coronary artery aneurysm with a fistulous connection to the right atrium mimicking a sinus of Valsalva aneurysm. Heart. 2003;89:E4.

4. Merchan A, Lopez-Minguez JR, Alonso F, Fernandez De La Concha J, Gonzalez R, Martinez De La Concha L. Giant left main coronary aneurysm without associated coronary lesions. Rev Esp Cardiol. 2002; 55:308-11.

5. Barettella MB, Bott-Silverman C. Coronary artery aneurysm: an unusual case report and a review of the literature. Cathet Cardiovasc Diagn. 1993;29:57-61.

\title{
Implantation of an autologously endothelialized homograft
}

\author{
Helmut Gulbins, MD, Angelika Goldemund, Antje Uhlig, Anita Pritisanac, MD, Bruno Meiser, MD, and \\ Bruno Reichart, MD, Munich, Germany
}

A

lthough cells have been shown to retain viability during cryopreservation, the luminal surface of cryopreserved homografts is normally not covered by viable endothelial cells. Therefore the surface of these grafts does not possess anticoagulatory properties. The low incidence of thromboembolic events in clinical settings could be explained by the high flow through the aortic valve and the resulting short contact times. Additionally, surviving donor cells elicit an immunologic reaction of the recipient. ${ }^{1-3}$ In experimental studies a chronic rejection of the homografts by the recipient was shown to be one major reason for graft degeneration and failure. Covering the luminal surface with autologous cells of the recipient might overcome these obstacles. This would lead to a physiologic luminal surface, resulting in an even further lowered thromboembolic risk. Additionally, the bloodstream would only come into contact with autologous cells, thus avoiding immunologic activation and subsequent rejection. We report the first, to our knowledge, clinical case of implantation of an autologously endothelialized aortic homograft. The study was approved by the local ethics committee, and the patient provided informed consent to participate.

\footnotetext{
From the Department of Cardiac Surgery, University Hospital Grosshadern, Munich, Germany.

Received for publication Feb 21, 2003; accepted for publication March 6, 2003.

Address for reprints: Helmut Gulbins, MD, Department of Cardiac Surgery, University Hospital Grosshadern, LMU Munich, D-81366, Munich, Germany (E-mail: H.Gulbins@ @hch.med.uni-muenchen.de).

J Thorac Cardiovasc Surg 2003;126:890-1

Copyright $\odot 2003$ by The American Association for Thoracic Surgery $0022-5223 / 2003 \$ 30.00+0$

doi:10.1016/S0022-5223(03)00609-3
}

\section{Clinical Summary}

A 59-year-old woman (blood group A) was referred to our hospital because of aortic valve stenosis on the basis of a bicuspid aortic valve. Preoperative examinations excluded significant coronary artery disease and other relevant concomitant diseases. Echocardiography showed an aortic valve stenosis with a calculated valvular orifice of $0.7 \mathrm{~cm}^{2}$, trivial aortic regurgitation, normal left ventricular function with mild hyper-

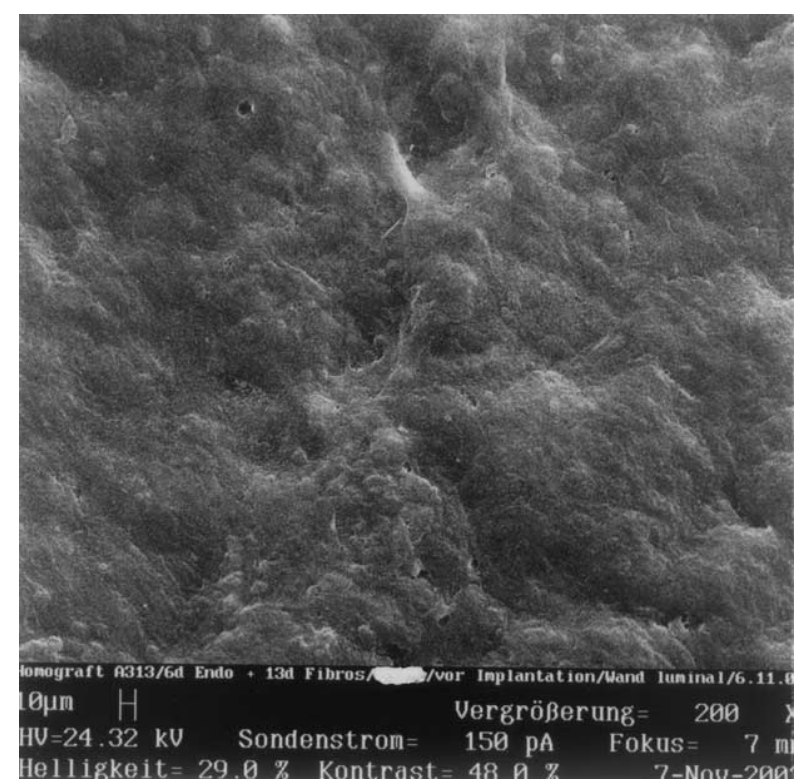

Figure 1. Scanning electron microscopic image. (Original magnification 200x.) A confluent endothelial cell layer is seen on the luminal surface. This specimen was taken directly before implantation. 